\title{
Sir2B, A Sirtuin Family Protein, Involves in Cell Adhesion Molecule Expression During Early Dictyostelium Development Upon Starvation
}

\author{
Hideo Taniura ", Shuhei Soeda, Ai Fujii, Mio Morioka, Minori Nakamura, Yui Sano \\ Laboratory of Neurochemistry, College of Pharmacy, Ritsumeikan University, Kusatsu, Japan \\ Email address: \\ hideo@fc.ritsumei.ac.jp (H. Taniura) \\ ${ }^{*}$ Corresponding author \\ To cite this article: \\ Hideo Taniura, Shuhei Soeda, Ai Fujii, Mio Morioka, Minori Nakamura, Yui Sano. Sir2B, A Sirtuin Family Protein, Involves in Cell Adhesion \\ Molecule Expression During Early Dictyostelium Development Upon Starvation. Cell Biology. Vol. 9, No. 1, 2021, pp. 1-6. \\ doi: $10.11648 /$ j.cb.20210901.11
}

Received: January 4, 2021; Accepted: January 18, 2021; Published: January 25, 2021

\begin{abstract}
Sirtuin involves in cellular processes to adapt to starvation in the endocrine and metabolic systems. We examined Sir2B function during early Dictyostelium development upon starvation. GFP-tagged Sir2B was expressed and the immunoreactivity was detected a punctate pattern near cell surface. Sir2B mRNA was expressed in vegetative cells and peaked at $2 \mathrm{~h}$ after starvation. Ectopic expression of Sir2B enhanced aggregate formation with increased expressions of cell adhesion molecules such as cadA, csaA, TgrB1, and TgrC1 at $8 \mathrm{~h}$ compared to those in control cells. Sir2B null-mutant cells (Sir2B KO cells) were generated and they formed aggregates smaller than those of wild-type cells after $24 \mathrm{~h}$. Because cell-cell adhesion affects aggregation size, cell cohesion assay of Sir2B KO cells showed cell-cell adhesion impairment at 8 and $10 \mathrm{~h}$ during development, and the expressions of four cell adhesion molecules were reduced in Sir2B KO cells compared to wild-type cells until $8 \mathrm{~h}$ after starvation. When RNAi-mediated cadA or csaA knockdown cells (cadA KD or csaA KD cells) were generated, csaA KD cells formed smaller aggregates and affected the expressions of other cell adhesion molecules to reduce, but cadA KD cells did not show to affect aggregation size and cell adhesion molecules expressions apparently. These results suggest that Sir2B is involved in csaA expression and affects other cell adhesion molecules during early development upon starvation.
\end{abstract}

Keywords: Sirtuin, Sir2B, Cell Adhesion, Development

\section{Introduction}

Sirtuin is originally discovered in Saccharomyces cerevisiae as Sir2, and Sir2 orthologs are highly conserved throughout evolution from archaebacteria to eukaryote. Sirtuin exhibits various enzymatic activities such as protein deacetylase and ADP ribosyltransferase. These enzymatic activities are generally activated in response to calorie restriction by the utilization of nicotine adenine dinucleotide $\left(\mathrm{NAD}^{+}\right)$as the co-substrate. SIRT1 among 7 mammalian Sirtuin is well-characterised with respect to its physiological functions. SIRT1 interacts with many regulatory proteins such as forkhead box protein O1, peroxisome proliferator-activated receptor, peroxisome proliferator-activated receptor gamma coacivator 1-alpha and STAT5 to adapt to starvation in the endocrine and metabolic systems [1-4].

The cellular slime mold, Dictyostelium discoideum, is a model organism for studying the molecular mechanisms of regulating cellular developmental process. Dictyostelium cells is a eukaryote and grow vegetatively. Upon starvation, they start to gather with neighboring cells to form aggregates consisting of up to $10^{5}$ cells. Because Dictyostelium cells start development in response to starvation, Sirtuin activation may be important for developmental process. Dictyostelium discoideum encodes at least 5 Sirtuins, Sir2A-Sir2E, that show sequence similarity to human Sirtuin. We have previously reported that Sirtuin activator resveratrol and inhibitor nicotinamide affect early development [5] and Sir2D regulates adenylate cyclase (aca) expression through interaction with a putative transcription factor MybB [6]. Katayama et al. have reported that Sir2A null mutant is less 
sensitive to starvation than is the wild-type cells [7]. mTOR is also known as a key player in metabolic stress management, and Sirtuin as well as mTOR have been linked with the lifespan extension upon calorie restriction in model organisms $[8,9]$. mTOR1 has a role as a nutrient sensor and mTOR2 regulates aca activation in controlling aggregation in Dictyostelium $[10,11]$.

Here, we examined roles of Sir2B during early development and found that Sir2B was involved in cell-cell adhesion molecules expression during early Dictyostelium development upon starvation. Cell-cell adhesion mediates cell gathering during aggregate formation. Four cell adhesion molecules such as cadA, csaA, TgrB1, and TgrC1 are expressed during early development $[12,13]$. cadA sequence has some homology with the extracellular domain of cadherins. csaA is a globular protein of the immunoglobulin superfamily and has a similarity to the neural cell adhesion molecule N-CAM. TgrB1 and TgrC1 are unique proteins to mediate cell-cell adhesion through heterophilic interaction, which have no homology with other molecules in higher organism. cadA and csaA expression coincide with the onset of aggregation, while TgrB1 and $\mathrm{TgrC} 1$ are initiated at mid-aggregation stage [13].

\section{Materials and Methods}

\subsection{Growth and Development of Dictyostelium Ax-2 Cells}

Dictyostelium discoideum Ax-2 cells were provided by the National BioResources Project of Japan. Ax-2 cells were grown in HL5 medium consisting of peptone, yeast extract, $\mathrm{Na}_{2} \mathrm{HPO}_{4}, \mathrm{KH}_{2} \mathrm{PO}_{4}$, glucose, and folate [14]. Ax-2 cells were developed under submerged culture in Na-K phosphate buffer (10 mmol/L Na-K phosphate buffer, $20 \mathrm{mmol} / \mathrm{L} \mathrm{MgSO}_{4}$, and 2 $\mathrm{mmol} / \mathrm{L} \mathrm{CaCl}_{2}, \mathrm{pH} 6.1$ ) over $24 \mathrm{~h}$.

\subsection{Cloning and Expression of Sir2B}

The entire coding sequence of Sir2B (DDB_G0286671) was amplified from cDNA and cloned into pBluescript using the following primers: 5 '- tgcggatccatgattaatatattaaaggac-3' and 5' - gcgetcgagttaaaatgaattaaatagagt-3'. GFP-tagged Sir2B at $\mathrm{N}$ terminus was expressed using pTX-GFP expression vector [15]. The construct was introduced into Ax-2 cells by electroporation and stable transformants were selected with G418. Control cells were generated by introducing empty vector without GFP. For fluorescence immunocytochemistry, the cells were fixed in $4 \%$ formaldehyde for $20 \mathrm{~min}$, permeabilized with methanol, and incubated with anti-GFP antibody (Invitrogen). The cells were also stained with 4'6'-diamidino-2-phenylindole dihydrochloride (DAPI, Life Technologies) to visualize nuclei. The images were obtained using a fluorescence microscope (EVOS, ThermoFisher Scientific).

\subsection{Quantitative Reverse Transcription-PCR (qRT-PCR)}

Total RNA was extracted, and cDNA was synthesized from vegetative, and 4-, 6-, and 8-h starved Sir2B expressing cells under submerged culture using TRI reagent (Sigma-Aldrich). RT-PCR products were quantified by LightCycler instrument (Roche Diagnostics). Melting curves were analyzed to confirm a single species of each PCR product. Actin 15 was used as an internal standard to quantify the relative expression of each cDNA. Statistical significance was tested using Student's t-test. The following primers were used for RT-PCR. actin 15; 5'-taaatccaaaagccaacagag-3' and 5'-ttggaaagttgagagtgaagc-3', aca; 5'-ttgcaggtttccaagaata-3' and 5'-tttgctctactgataccgat-3', carA; 5'-taaatatgtttccaccagca-3' and 5'-taccacgacttgaactatatg-3', Sir2B; 5'-tagtttatccattcgcttc-3' and 5'-tatatctctattacctcttgc-3', TgrB1; 5'-tagcattaatttcatttgecg-3' and 5'-aggtacttttatctggetttg-3', TgrC1; 5'-ttgtttatcatgccattccg-3' and 5'-ttttaaagtaataggcaagag-3', cadA; 5'-cttaccaggagcttttcaatg-3' and 5'-atggctaacaatttcagaatc-3', and csaA; 5'-ctccggttataaatagtgtca-3' and 5'-ttgtggcttcagttttaata-3'.

\subsection{Isolation of Sir2B-null Mutant (Sir2B KO Cells)}

The Sir2B-null mutant was generated by gene targeting. The targeting construct contains a genomic fragment of Sir2B coding region from the position +75 to $+2515(+1$ at the beginning of the ATG start codon) in which a BSR cassette was inserted at the HindIII site (the position +1445$)$. The following primers were used for PCR amplification: 5'-tgcgtcgacggcaataaaaaactcttatcaac-3', and 5'-gcgactagttatctctattacetcttgc-3'. The construct was linearized with SalI and SpeI and introduced into Ax-2 cells. Transformants were selected for blasticidin resistance. Homologous recombination was confirmed by genomic PCR. Two sets of primers (Wt-F/BSR-R and BSR-F/Wt-R) recognize part of the region that is not present in the targeting construct (Wt-F and Wt-R) and in BSR cassette (BSR-F and BSR-R) (Figure 3A). The primers used were as follows: Wt-F: 5'-ttttatttgggttgggcaat-3', BSR-R: 5'-attgtaatcttctctgtcgcta-3', BSR-F: 5'-ttttgacacgattgtagctg-3', and Wt-R: 5'-agtattgaaataatcaacacct-3'.

\subsection{Cell Cohesion Assay}

Cell cohesion assay was performed according to Roisin-Bouffay et. al. [12]. Cell samples were collected at 6, 8, and $10 \mathrm{~h}$ after starvation and vortexed for $20 \mathrm{~s}$. The number of unaggregated cells (singlets and doublets) in these samples were determined using a hemocytometer and the percentage of cells that had been recruited into aggregates was estimated. Statistical significance was tested using Student's t-test.

\subsection{RNA Interference (RNAi) Mediated Knockdown Mutant of cadA or csaA (cadA KD or csaA KD Cells)}

RNAi-mediated gene silencing has been established in Dictyostelium [16]. We constructed expression vectors to express a stem-loop RNA against cadA or csaA mRNA to knockdown the expression. One fragment containing nucleotide +34 to +566 of the coding region of cadA $(+1$ at the beginning of the ATG start codon) was cloned in sense orientation into pTX BamHI/XhoI sites. Another fragment 
containing nucleotide +35 to +417 was fused in antisense orientation into $\mathrm{pTX} \mathrm{XbaI} / \mathrm{XhoI}$ sites. One fragment containing nucleotide +32 to +1497 of the coding region of csaA was cloned in sense orientation into pTX BamHI/XhoI sites. Another fragment containing nucleotide +32 to +1069 was fused in antisense orientation into $\mathrm{pTX} \mathrm{XbaI/XhoI} \mathrm{sites.}$ The cadA or csaA RNAi vector was introduced into Ax-2 cells and stable transformants were selected with G418. Control cells were generated by introducing empty vector. qRT-PCR was performed to quantify the levels of cadA or csaA mRNA expression. Actin 15 level was used as an internal standard. Statistical significance was tested using Student's t-test.

A

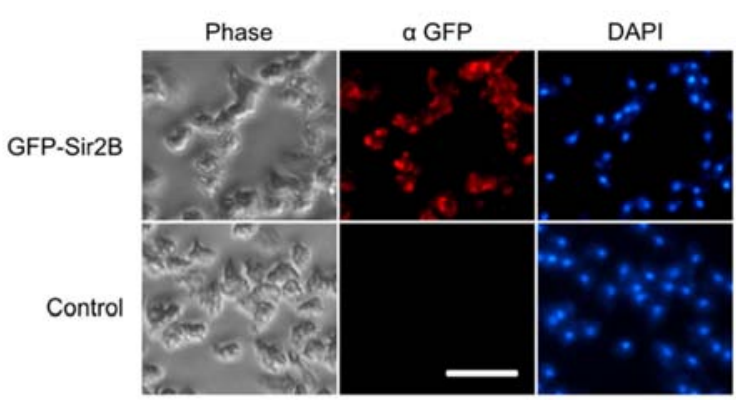

B

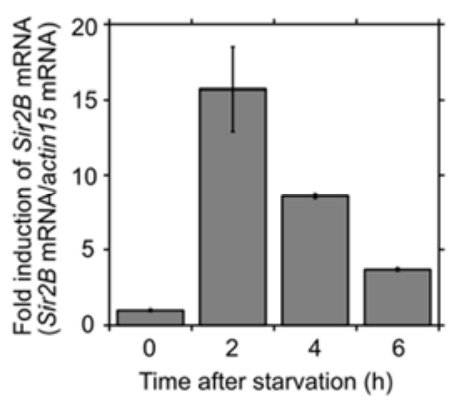

C

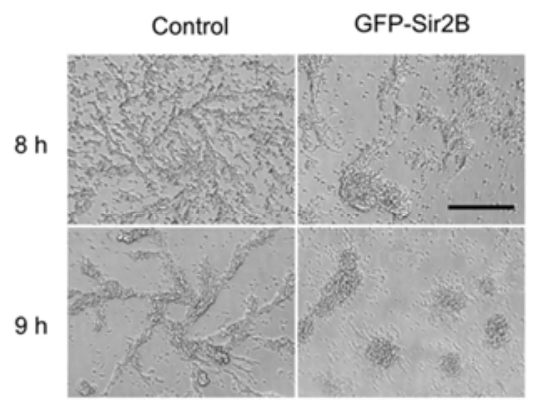

Figure 1. Development of Sir2B expressing cells. A. Localization of GFP-Sir2B in axenically grown Ax-2 cells. Expression of GFP-Sir2B was detected using anti-GFP antibody under a fluorescent microscope (middle panel, $\alpha$ GFP). Empty vector without GFP transfected cells were used as a control. The same cells were also stained with DAPI to show the nuclei (right panel, DAPI). The left panel shows the phase-contrast image of the same field (left panel, Phase). Scale bar: $50 \mu m$. B. mRNA expression of Sir2B during early development. Total RNA was extracted from the cells at 0, 2, 4, and $6 \mathrm{~h}$ after starvation. qRT-PCR was performed using specific primers for Sir $2 B$ or actin 15. The quantified results were normalized against actin $15 \mathrm{mRNA}$ levels. Each value represents the mean \pm standard error (SE) $(n=3)$. C. Control (empty vector) and Sir2B expressing cells were developed under submerged culture. Development was imaged at 8 and 9 h. Scale bar: $200 \mu \mathrm{m}$.

\section{Results}

\subsection{Sir2B in Early Development}

We expressed Sir2B protein carrying GFP-tag at N terminus (GFP-Sir2B) and examined subcellular localization. As shown in Figure 1A, GFP-Sir2B immunoreactivity was detected as a punctate pattern near peripheral membrane and was not coincide with DAPI nuclear staining. Endogenous Sir2B mRNA was expressed in vegetative cells and the expression was peaked at $2 \mathrm{~h}$ after starvation by 15.7-fold (Figure 1B). When Sir2B expressing cells were developed under submerged condition, the initiation of streaming formation was almost the same timing as that of control cells, but enhanced aggregate formation was observed (Figure 1C). Cells formed streaming transiently and gathered rapidly to form aggregates.
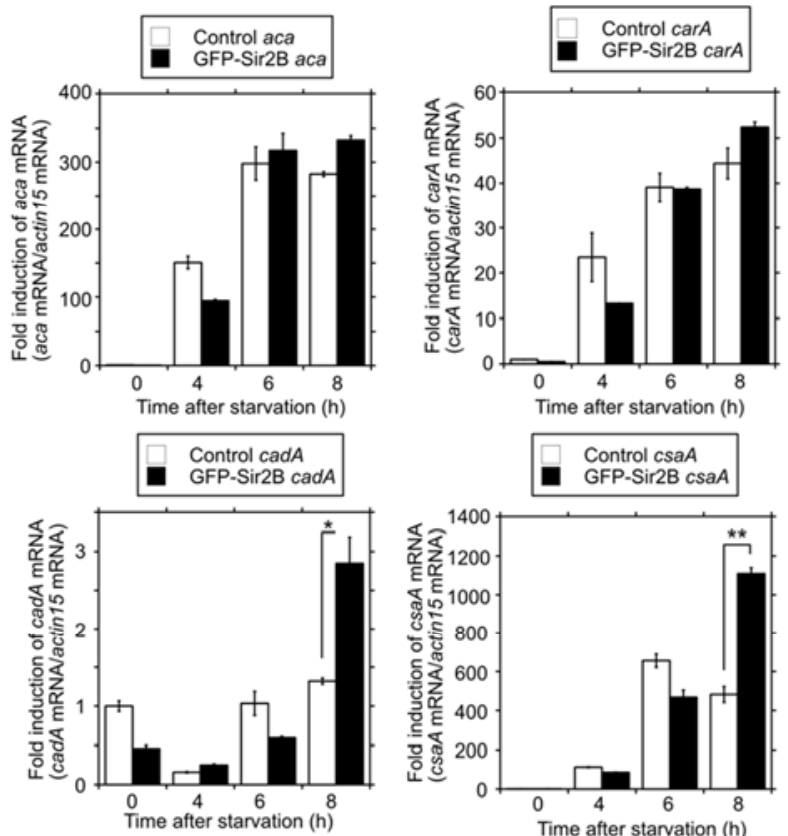

Control TgrB1
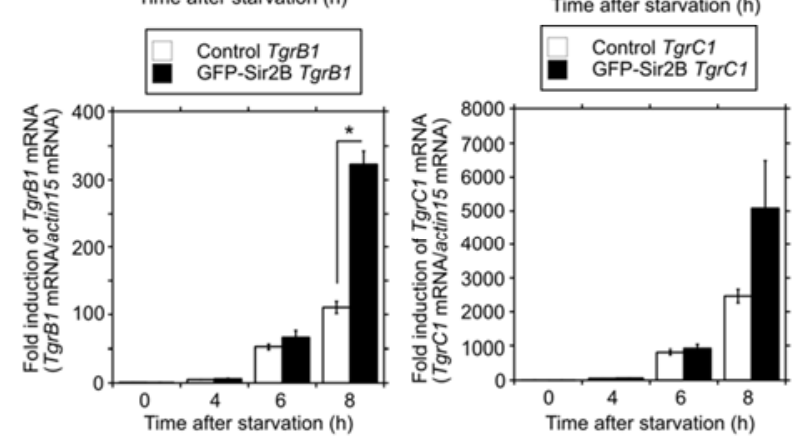

Figure 2. Alteration of $m R N A$ expression of aca, carA, and cell adhesion molecules in Sir2B expressing cells during early development. Control and Sir $2 B$ expressing cells were developed under submerged culture. Total RNA was extracted from the cells at 0, 4, 6, and $8 \mathrm{~h}$ after starvation. qRT-PCR was performed using specific primers for aca, carA, cadA, csaA, TgrB1, $\operatorname{TgrC1}$, or actin 15. The quantified results were normalized against actin 15 $m R N A$ levels. Each value represents the mean \pm standard error (SE) $(n=3)$. Asterisk indicates a significantly different value $(* P<0.05$ and $* * P<0.01)$ from the control's value. 
We examined several genes expression during early development of GFP-Sir2B expressing cells. Early genes such as aca and carA mRNA expression levels were not altered between Sir2B expressing and control cells until $8 \mathrm{~h}$ (Figure 2, aca and carA). Because of enhanced aggregate formation of GFP-Sir2B cells, we next examined cell adhesion molecules expression. cadA expression was relatively high in vegetative state and decreased at $4 \mathrm{~h}$, then the expression was increased at 6 and $8 \mathrm{~h}$ in control cells. Sir2B expression significantly increased the expression of cadA by 2.16 -fold compared to control cells at $8 \mathrm{~h}$ (Figure 2, cadA). csaA was also induced at 4 and $6 \mathrm{~h}$ in control cells, and Sir2B expression significantly increased the expression of csaA at $8 \mathrm{~h}$ by 2.3 -fold compared to control cells. TgrB1 and TgrC1 mediate cell-cell adhesion via heterophilic interaction [17]. Both of TgrB1 and TgrC1 were induced at 6 and $8 \mathrm{~h}$ in control cells, and Sir2B expression significantly increased the expression of TgrB1 at 8 h by 2.92 -fold compared to control cells. TgrC1 expression was also increased at $8 \mathrm{~h}$ (Figure 2, TgrB1 and TgrC1).

\subsection{Sir2B-null Mutant (Sir2B KO Cells)}

Next, we isolated Sir2B-null mutant by conventional gene targeting. The targeting construct contains a genomic fragment of Sir2B coding region from the position +75 to +2515 ( +1 at the beginning of the ATG start codon) in which a BSR cassette was inserted at the HindIII site (the position +1445 ) (Figure 3A). The recombinant clone was identified by the presence of PCR products using two sets of primers shown (Figure 3A and B). The disruption of the Sir2B gene was further confirmed by qRT-PCR, and the expression level of Sir2B mRNA in Sir2B KO cells was reduced to $1.43 \%$ of that in wild-type cells (Figure 3C). To examine the development of Sir2B KO cells, we observed the starved cells under submerged condition. Sir2B KO cells showed negligible difference of development compared to wild-type cells but formed aggregates smaller than those of wild-type cells after $24 \mathrm{~h}$ (Figure 3D). This smaller aggregate formation was partially restored in Sir2B KO cells by GFP-Sir2B expression. The quantified results were also shown in Figure 3D.

Because low cell-cell adhesion results in aggregation streams breaking up into small groups [12], we examined whether Sir2B KO cells have altered cell-cell adhesion. Cells were starved for various times, and cell-cell adhesion was measured following Raisin-Bouffay et. al. [12]. As shown in Figure 4A, the adhesion of wild-type cells was initially low and then increased at 8 and $10 \mathrm{~h}$ after starvation. The adhesion of Sir2B KO cells was $21.1 \%$ against $70 \%$ of wild-type cells at $8 \mathrm{~h}$. Low adhesion of Sir2B KO cells was partially restored by GFP-Sir2B expression to $45.1 \%$ at $8 \mathrm{~h}$. We then examined mRNA expression levels of four cell adhesion molecules during early development. All the expressions of four cell adhesion molecules such as cadA, csaA, TgrB1, and TgrC1 were reduced in Sir2B KO cells compared to wild-type cells during early development until $8 \mathrm{~h}$ after starvation, and the reduction was restored by GFP-Sir2B expression (Figure 4B). cad A expression was reduced in Sir2B KO cells to about $12-18 \%$ of those in wild-type cells and restored by GFP-Sir2B expression at 6 and $8 \mathrm{~h}$. csaA expression levels were reduced in Sir2B KO cells to about $10 \%$ at 4 and $6 \mathrm{~h}$ and $50 \%$ at $8 \mathrm{~h}$ and restored by GFP-Sir2B expression at 6 and 8 h. TgrB1 expression levels were reduced to about $30-40 \%$ at 6 and $8 \mathrm{~h}$ and restored by GFP-Sir2B expression. TgrC1 expression levels were also reduced in Sir2B KO cells to $12 \%$ at $6 \mathrm{~h}$ and $25 \%$ at $8 \mathrm{~h}$ restored by GFP-Sir2B expression.
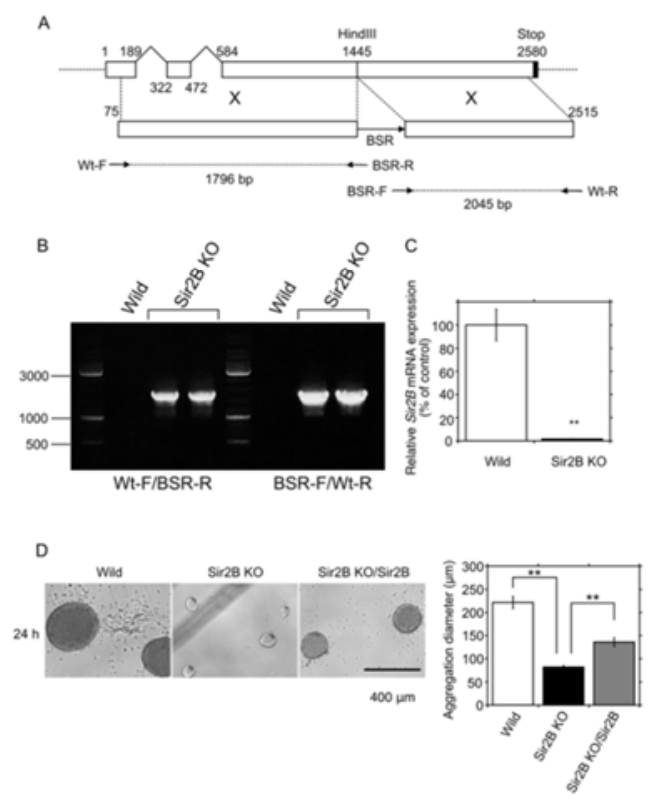

Figure 3. Preparation of Sir2B knockout cells (Sir2B KO cells). A Targeting construction. The targeting construct contains a genomic fragment that encompasses the Sir $2 B$ coding region from the position +75 to $+2515(+1$ at the beginning of the ATG start codon) in which a BSR cassette was inserted at the HindIII site (the position +1445). The Arrows denote two primer sets (Wt-F/BSR-R and BSR-F/Wt-R) for PCR analysis and estimated product size was also shown. B. PCR analysis. Genomic DNA was isolated from wild-type and Sir $2 B$ KO cells (Wild and Sir $2 B K O$ ). PCR analysis was performed using two primer sets of $W t-F / B S R-R$ and $B S R-F / W t-R$. Two primers of $W t-F$ and $W t-R$ recognize part of the region that is not present in the targeting construct and BSR-F and BSR-R are in the BSR cassette. C. qRT-PCR analysis of Sir $2 B$ $K O$ cells (Sir2B KO). Total RNA extracted from vegetative wild-type and Sir2B KO cells were used for oligo (dT)-primed qRT-PCR. The quantified results were normalized against actin $15 \mathrm{mRNA}$ levels. Each value represents the mean \pm standard error $(S E)(n=3)$. Asterisk indicates a significantly different value $(* * P<0.01)$ from the control's value. D. Aggregation size. Wild-type, Sir $2 B \mathrm{KO}$ and Sir2B KO expressed with GFP-Sir2B (Sir2B $K O / S i r 2 B)$ cells were developed under submerged culture. After $24 \mathrm{~h}$, long diameter of each aggregates formed was measured. Each value represents the mean \pm standard error (SE) (Wild, $n=17$; Sir $2 B \mathrm{KO}, n=31$; $\operatorname{Sir} 2 B \mathrm{KO} / \operatorname{Sir} 2 B$, $n=19)$. Asterisk indicates a significant different value $(* * P<0.01)$.

\subsection{RNAi-mediated Knockdown of cadA or csaA Expression (cadA KD or csaA KD Cells)}

The expressions of adhesion molecules may be influenced by each other. For example, the expression of csaA is dependent on cadA function and is reduced when cadA-mediated adhesion is blocked by EDTA, which argues that cadA-mediated cell contact is required for full csaA function [18]. We isolated cadA or csaA knockdown transformants (cadA or csaA KD cells) by RNAi and examined the expression levels of other adhesion molecules. The expression level of csaA or cadA mRNA in the RNAi expression vector transfected cells was reduced to $24 \%$ or $30 \%$ 
of empty vector-transfected control cells at $6 \mathrm{~h}$ or $0 \mathrm{~h}$ (vegetative cells) after starvation, respectively (Figure 5A). csaA KD cells formed aggregates smaller than those of control cells after $24 \mathrm{~h}$, but cadA KD cells did not under submerged culture (Figure 5B). When we examined cadA expression in csaA KD cells and csaA expression in cadA KD cells, csaA reduction affected cadA expression but cadA reduction did not affect csaA expression (Figure 5C, Top 2 panels). cadA mRNA was reduced to about $20 \%$ at 0,4 , and $6 \mathrm{~h}$ and $42.5 \%$ at $8 \mathrm{~h}$ in csaA KD cells compared with that in control cells. TgrB1 and TgrC1 expressions were also affected in csaA KD cells (Figure 5C, Bottom 2 panels). TgrB1 mRNA was reduced to $20-29 \%$ at 0,4 , and $6 \mathrm{~h}$ in csaA KD cells compared with that in control cells. TgrB1 was also reduced moderately to $46.7 \%$ in cadA KD cells, but it is not significant. TgrC1 was reduced to $20 \%$ at 0,4 , and 6 $\mathrm{h}$ in csaA KD cells, but it is not significant. These results suggest that Sir2B may be involved in csaA expression, and expression of other adhesion molecules are affected by csaA.
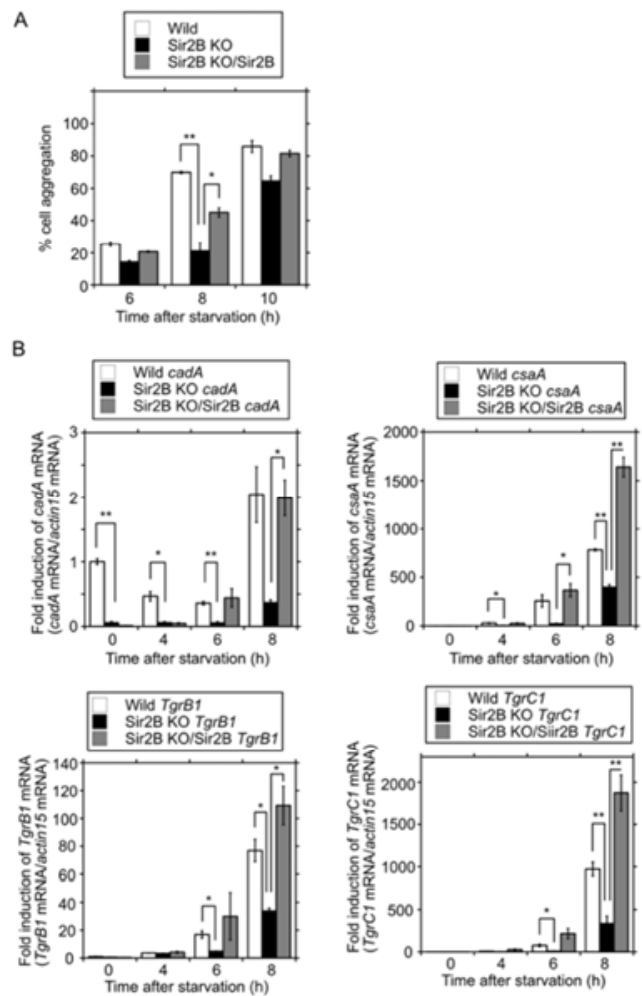

Figure 4. Alteration of $m R N A$ expression of cell adhesion molecules in Sir $2 B$ $K O$ cells during early development. A. Cell cohesion assay. Wild-type, Sir $2 B$ $K O$ and Sir $2 B K O$ expressed with GFP-Sir $2 B$ (Sir $2 B$ KO/Sir2B) cells were developed at $2 \times 10^{6}$ cells $/ \mathrm{ml}$ in $\mathrm{Na}-\mathrm{K}$ phosphate buffer. Cell samples were collected at 6,8 , and $10 \mathrm{~h}$ after starvation and vortexed for $20 \mathrm{~s}$. The number of unaggregated cells (singlets and doublets) was determined using a hemocytometer at 6, 8, and $10 \mathrm{~h}$ and the percentage of cells that had been recruited into aggregates was estimated. Each value represents the mean \pm standard error $(S E)(n=3)$. Asterisk indicates a significantly different value $(* P<0.05$ and $* * P<0.01)$. B. Cell adhesion molecules expression. Wild-type, Sir $2 B$ KO and Sir $2 B$ KO expressed with GFP-Sir2B (Sir $2 B$ KO/Sir2B) cells were developed under submerged culture. Total RNA was extracted from the cells 0, 4, 6 and 8 h after starvation. qRT-PCR was performed using specific primers for TgrB1, TgrC1, cadA, csaA or actin 15. Each value represents the mean \pm standard error $(S E)(n=3)$. Asterisk indicates a significantly different value $(* P<0.05$ and $* * P<0.01)$ from the control's value.

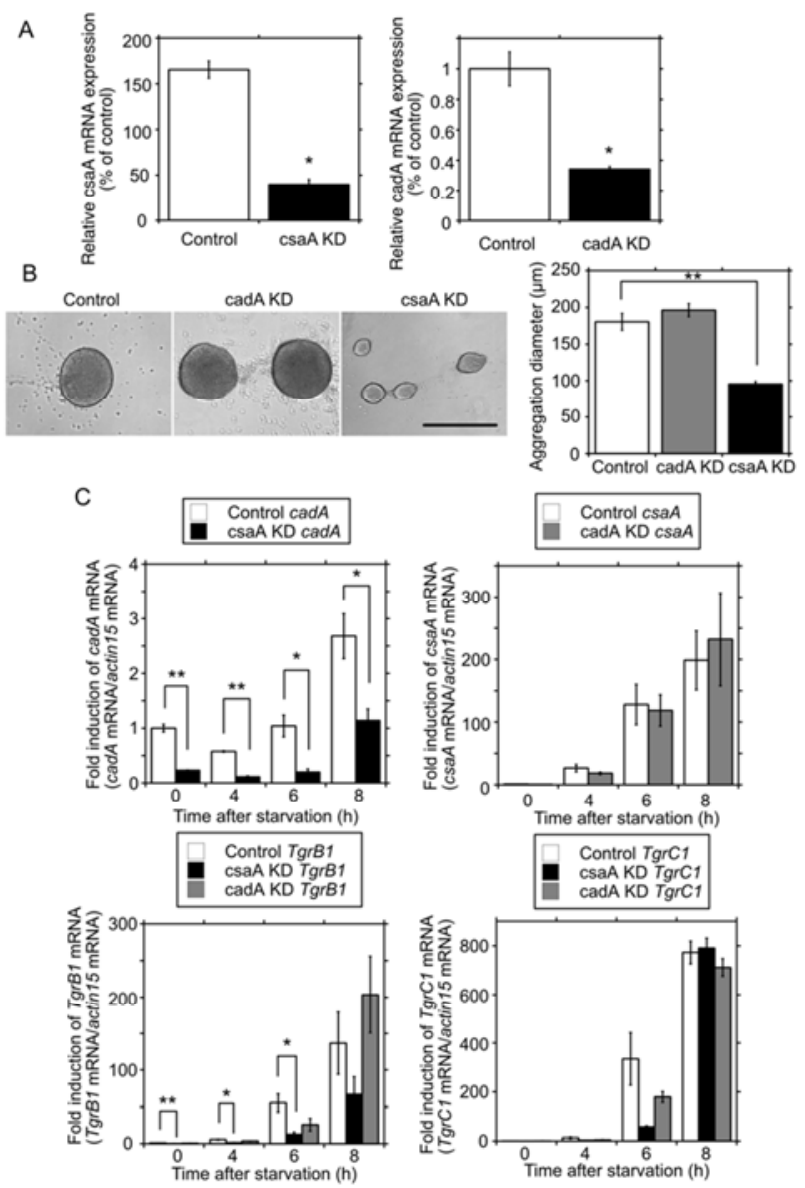

Figure 5. RNAi-mediated knockdown of cadA or csaA expression (cadA KD or csaA KD cells) A. qRT-PCR analysis of cadA or csaA KD cells. Total RNA extracted from vegetative control (empty vector) and cadA KD cells or control and csaA KD cells at $0 \mathrm{~h}$ (vegetative cells) or $6 \mathrm{~h}$ after starvation, respectively was used for oligo (dT)-primed qRT-PCR. The quantified results were normalized against actin $15 \mathrm{mRNA}$ levels. Each value represents the mean \pm standard error $(S E)(n=3)$. Asterisk indicates a significantly different value $(* P<0.05)$ from the control's value. B. Aggregates size. Control, cadA KD and csaA KD cells were developed under submerged culture. After $24 \mathrm{~h}$, long diameter of each aggregates formed was measured. Each value represents the mean \pm standard error (SE) (control, $n=23$; cadA KD, $n=45 ; \operatorname{csaAKD}$, $n=37)$. Asterisk indicates a significantly different value $(* * P<0.01)$. C. Cell adhesion molecules expression. Control, csaA KD and cadA KD cells were developed under submerged culture. Total RNA was extracted from the cells 0 , 4, 6 and 8 h after starvation. qRT-PCR was performed using specific primers for TgrB1, TgrC1, cadA, csaA or actin 15. The quantified results were normalized against actin $15 \mathrm{mRNA}$ levels. Each value represents the mean \pm standard error $(S E)(n=3)$. Asterisk indicates a significantly different value $(* P<0.05$ and $* * P<0.01)$ from the control's value.

\section{Discussion}

Sirtuin interacts with many regulatory proteins in response to calorie restriction. We have previously reported that Sir2D regulates aca expression through interaction with a putative transcription factor, MybB in early Dictyostelium development upon starvation $[6,19]$. Here, we examined roles of Sir2B during early development. While Sir2D is expressed in the nuclei, Sir2B was distributed a punctate expression pattern near peripheral membrane and was not coincide with DAPI nuclear staining. Sir2B may interact with a signaling molecule. Sir2B 
expressing cells did not accelerate the timing of streaming, consistent with no alteration of aca and carA expressions compared to control cells at early development [20]. However, Sir2B expression enhanced aggregate formation, and increased the expressions of cell adhesion molecules. While Sir2B KO cells formed smaller aggregates than those of wild-type cells with reduction of cell adhesion molecules expressions. smlA mutants also show reduced expression of cadA and csaA during streaming and early aggregation, leading to reduced intercellular adhesion and causes streams to break up and form aggregates smaller [12]. When we prepared cadA and csaA KD cells and examined the effect of cadA or csaA reduction on other cell adhesion molecules expressions. csaA reduction affected all other cell adhesion molecules expressions, but effects of cadA reduction were not apparent. Because we observed reduction of csaA and cadA, but not TgrB1 and TgrC1 in RNAi-mediated knockdown cells of Sir2B (data not shown), Sir2B may be primary involved in csaA expression, and expressions of other adhesion molecules are affected by csaA. Here, our findings suggest that Sir2B regulates the expression of cell-cell adhesion molecule during aggregate formation upon starvation. Further experiments are necessary to confirm the roles of Sir2B during early Dictyostelium development and to identify the target molecule to interact with.

\section{Conclusions}

Sir2B-null mutant cells impair the cell-cell adhesion and show decreased expressions of cell adhesion molecules during early development, and forms smaller aggregates. csaA but not cadA knockdown cells form smaller aggregates with the reduction of the expressions of other cell adhesion molecules. Therefore, csaA may be a primary target for Sir2B.

\section{Acknowledgements}

This work was supported in part by MEXT-Supported Program for the Strategic Research Foundation at Private Universities and a grant from Takeda Science Foundation.

\section{References}

[1] Picard, F., Kurtev, M., Chung, N., Topark-Ngarm, A., Senawong, T., de Oliveira, R. M., Leid, M., McBurney, M. W., Guarente, L. (2004) Sirtl promotes fat mobilization in white adipocytes by repressing PPAR- $\gamma$. Nature 429: 771-776.

[2] Michan, S., Sinclair, D. (2007) Sirtuins in mammals: insights into their biological function. Biochem. J. 404: 1-13.

[3] Vassilopoulos, A., Fritz, K. S., Petersen, D. R., Gius, D. (2011) The human sirtuin family: evolutionary divergences and functions. Hum. Genom. 5: 485-496.

[4] Yamamoto, M., Takahashi, Y. (2018) The essential role of SIRT1 in hypothalamic-pituitary axis. Front. Endocrinol. 9: 605 .

[5] Soeda, S., Taniura, H. (2018) Sirtuin activator and inhibitor affect early Dictyostelium development upon starvation. Cell Biol. 6: 13-19.

[6] Taniura, H., Soeda, S., Ohta, T., Oki, M., Tsuboi, R. (2019) Sir2D, a Sirtuin family protein, regulates adenylate cyclase A expression through interaction with the MybB transcription factor early in Dictyostelium development upon starvation. Heliyon 3: $\mathrm{e} 01301$.

[7] Katayama, T., Okuwa, T., Yasukawa, H. (2008) Difference in sensitivity to amino acid starvation between the wild type and Sir2A-null mutant of Dictyostelium. Jpn. J. Protozool. 41: 153-158.

[8] Saxton, R. A., Sabatini, D. M. (2017) mTOR signaling in growth, metabolism, and disease. Cell 168: 960-976.

[9] Kapahi, P., Kaeberlein, M., Hansen, K. (2017) Dietary restriction and lifespan: Lessons from invertebrate models. Ageing Res. Reviews 39: 3-14.

[10] Lee, S., Comer, F. I., Sasaki, A., McLeod, I. X., Duong, Y., Okumura, K., Yates III, J. R., Parent, C. A., Firtel, R. A. (2005) TOR complex 2 integrates cell movement during chemotaxis and signal relay in Dictyostelium. Mol. Biol. Cell 16: $4572-4583$.

[11] Jaiswal, P., Kimmel, A. (2019) mTORC1/AMPK responses define a core gene set for developmental cell fate switching. BMC Biol. 17: 58.

[12] Raisin-Bouffay, C., Jang, W., Caprette, D. R., Gomer, R. H. (2000) A precise group size in Dictyostelium is generated by a cell-counting factor modulating cell-cell adhesion. Mol. Cell 6: 953-959.

[13] Siu, C. H., Srikanthadevan, S., Wang, J., Hou, L., Chen, G., Xu, X., Thomson, A., Yang, C. (2011) Regulation of spatiotemporal expression of cell-cell adhesion molecules during development of Dictyostelium discoideum. Dev. Growth Differ. 53: 518-527.

[14] Taniura, H., Tanabe, N., Bando, Y., Arai, N. (2015) Nse1 and Nse4, subunits of the Smc5-Smc6 complex, are involved in Dictyostelium development upon starvation. Dev. Growth Differ. 57: 430-443.

[15] Levi, S., Polyakov, M., Egelhoff, T. T. (2000) Green fluorescent protein and epitope tag fusion vectors for Dictyostelium discoideum. Plasmid 44: 231-238.

[16] Martens, H., Novotny, J., Oberstrass, L., Steck, T. L., Postlethwait, P., Nellen, W. (2002) RNAi in Dictostelium: the role of RNA-directed RNA polymerase and double-stranded RNase. Mol. Biol. Cell 13: 445-453.

[17] Chen, G., Xu, X., Wu, X., Thomson, A., Siu, C-H. (2014) Assembly of the TgrB1-TgrC1 cell adhesion complex during Dictyostelium discoideum development. Biochem. J. 459: 241-249.

[18] Coates, J. C., Harwood, A. J. (2001) Cell-cell adhesion transduction during Dictyostelium development. J. Cell Sci. 114: 4349-4358.

[19] Otsuka, H., Van Haastert, P. J. M. (1998) A novel Myb homolog initiates Dictyostelium development by induction of adenylyl cyclase expression. Genes Dev. 12: 1738-1748.

[20] Schaap, P. (2016) Evolution of developmental signaling in Dictyostelid social amoebas. Curr. Opin. Genet. Dev. 39: 29-34. 